Journal of

Experimental

Social Psychology

Journal of Experimental Social Psychology 44 (2008) 1100-1105

www.elsevier.com/locate/jesp

\title{
Brief exposures: Male sexual orientation is accurately perceived at $50 \mathrm{~ms}$ is
}

\author{
Nicholas O. Rule *, Nalini Ambady \\ Tufts University, Department of Psychology, 490 Boston Avenue, Medford, MA 02155, USA
}

Received 4 January 2007; revised 27 November 2007

Available online 25 January 2008

\begin{abstract}
People have proved adept at categorizing others into social categories, at least when the categorical distinction is perceptually obvious (e.g., age, race, or gender). There remain many social groups whose boundaries are less clear, however. The current work therefore tested judgments of an ambiguous social category (male sexual orientation) from faces shown for durations between $33 \mathrm{~ms}$ and 10,000 ms. The sexual orientation of faces presented for $50 \mathrm{~ms}, 100 \mathrm{~ms}, 6500 \mathrm{~ms}, 10,000 \mathrm{~ms}$, and at a self-paced rate (averaging $1500 \mathrm{~ms}$ ), was categorized at above-chance levels with no decrease in accuracy for briefer exposures. Previous work showing impression formation at similar speeds relied on consensus to determine the validity of judgments. The present results extend these findings by providing a criterion for judgmental accuracy - actual group membership.
\end{abstract}

(C) 2007 Elsevier Inc. All rights reserved.

Keywords: Person perception; Judgmental accuracy; Sexual orientation; Nonverbal behavior; Social cognition

As we navigate the social world, we are constantly called upon to make judgments of those around us: friend or foe, approach or avoid, mate with or fight with, etc. As such, we are consistently making snap judgments of those we encounter (see Ambady \& Rosenthal, 1992). We categorize others into groups (Macrae \& Bodenhausen, 2000), infer their intentions (Mason, Tatkow, \& Macrae, 2005), and make judgments about their character (Berry \& McArthur, 1985), to name a few.

Whereas perceptually obvious social categories such as age (Wright \& Stroud, 2002), race (Richeson \& Trawalter, 2005), or gender (Macrae \& Martin, 2007) are quickly and efficiently perceived based on facial cues, little work has investigated the perceptibility of more ambiguous social

\footnotetext{
We would like to thank Max Weisbuch and Reg Adams for their advice and assistance throughout the execution of this study. This research was supported in part by a National Science Foundation Graduate Research Fellowship to Nicholas O. Rule and a National Science Foundation research grant (NSF BCS-0435547) to Nalini Ambady.

* Corresponding author. Fax: +1 6176273181.

E-mail address: nicholas.rule@tufts.edu (N.O. Rule).
}

groups. This leaves open the question as to whether groups that lack clearly defined visual markers can be accurately perceived from brief glimpses of the face.

One hallmark of person construal judgments is the speed with which they can occur. Subliminal presentations of the faces of perceptually obvious groups have long shown evidence of accurate and efficient categorization (e.g., Eberhardt, Goff, Purdie, \& Davies, 2004). Recently, however, these processes have been demonstrated for less evident aspects of identity. Bar, Neta, and Linz (2006) demonstrated that threat in faces was consistently detected at $39 \mathrm{~ms}$ and $1700 \mathrm{~ms}$ exposure times but that judgments of intelligence were not consistent at such rapid speeds. Willis and Todorov (2006), however, showed that judgments of personality traits (i.e., aggressiveness, competence, likeability, and trustworthiness) and attractiveness were consistent between $100 \mathrm{~ms}$ and $1000 \mathrm{~ms}$. Both these studies looked exclusively at consensual judgments, or the agreement among raters. But there has been no work, to our knowledge, about whether such judgments are accurate. The current study therefore attempts to assess whether rapid expo- 
sures to faces will allow for accurate judgments of an ambiguous social identity: male sexual orientation.

To date, limited evidence has suggested that sexual orientation can be accurately discerned from thin slices, or brief observations of dynamic nonverbal behavior (as short as 1000 ms; Ambady, Hallahan, \& Conner, 1999), and paralinguistic cues from the voice (e.g., Gaudio, 1994; Smyth, Jacobs, \& Rogers, 2003). Self-report data have also shown that individuals believe sexual orientation can be judged from nonverbal information (Shelp, 2002) but have been restricted to descriptions of intentional actions (such as eye gaze; Carroll \& Gilroy, 2002; Nicholas, 2004), rather than examining unintentional factors, such as appearance or non-conscious behaviors. In the current work we examine whether male sexual orientation can be judged from static, exclusively facial cues at rapid exposures.

\section{Study 1A}

\section{Method}

\section{Participants}

Ninety undergraduates ( $n=68$ females) were assigned to six conditions ( $n=15$ per condition) based on exposure time: $33 \mathrm{~ms}, 50 \mathrm{~ms}, 100 \mathrm{~ms}, 6500 \mathrm{~ms}, 10,000 \mathrm{~ms}$, and selfpaced judgments.

\section{Stimuli}

Images were obtained from public, online personal advertisements posted for use in various major cities across the U.S. Men in the images were therefore self-defined for sexual orientation, were anonymous, and were available to the public domain. Images were taken from the 18 to 30 age group on the personals websites. Only photos of headshots were downloaded for use. Although hundreds of images were initially collected, only those images presenting a directly oriented face free of any facial alterations (such as jewelry, glasses, or facial hair) were selected, resulting in 45 homosexual and 45 heterosexual images. The images were removed from their original context and placed onto a white background. Targets' ears and hair were retained in the cropping while all other extra-facial information (e.g., neck) was removed. Images were then gray-scaled and standardized to $3^{\prime \prime} \times 5^{\prime \prime}$ dimensions. To preserve anonymity and respect privacy, none of the targets' sexual orientations were disclosed to participants. Further, none of the photos were obtained from the local geographic area.

\section{Procedure}

Study 1A consisted of six conditions based on the presentation time of the photographs. Participants made judgments based on faces they saw for either $33 \mathrm{~ms}, 50 \mathrm{~ms}$, $100 \mathrm{~ms}, 6500 \mathrm{~ms}, 10,000 \mathrm{~ms}$, or at their own self-paced rate (i.e., photos were presented until the participant pressed a response key).
Participants were instructed that they would be seeing men's faces on the computer screen and that they would be asked to classify via key-press whether these targets were likely to be either gay or straight. In the $33 \mathrm{~ms}, 50 \mathrm{~ms}$, and $100 \mathrm{~ms}$ conditions, participants were given several practice trials to acquaint them with the speed of presentation of the faces. No feedback was given during any of these trials, nor were these faces presented in the actual experiment.

Each face was presented in random order. Participants had $1000 \mathrm{~ms}$ rest, saw the photo for the respective exposure time, and were then prompted to make a dichotomous judgment of the target's probable sexual orientation. In the $33 \mathrm{~ms}, 50 \mathrm{~ms}$, and $100 \mathrm{~ms}$ conditions, photos were preceded by a $1000 \mathrm{~ms}$ fixation cross and succeeded by a 100 ms mask, which consisted of a scrambled face matched for high and low spatial frequencies. Participants were not asked to volunteer their own sexual orientation.

\section{Results}

Sexual orientation was judged from male faces at levels significantly greater than chance in all but the $33 \mathrm{~ms}$ condition. Accuracy scores were calculated for each participant by dividing the number of correct classifications by the total number of images. $T$-Tests were then used to compare the accuracy scores against chance (.5) for each condition: (a) $10,000 \mathrm{~ms} \quad\left[M_{\text {accuracy }}=60 \% ; t(14)=5.43, p<.001\right.$, $r=.82], \quad$ (b) $6500 \mathrm{~ms} \quad\left[M_{\text {accuracy }}=58 \% ; \quad t(14)=5.22\right.$, $p<.001, r=.81]$, (c) self-paced ${ }^{1}\left[M_{\text {accuracy }}=62 \% ; t(14)=\right.$ $6.47, p<.001, r=.87], \quad(\mathrm{d}) 100 \mathrm{~ms} \quad\left[M_{\text {accuracy }}=62 \%\right.$; $t(14)=7.74, p<.001, r=.90]$, (e) $50 \mathrm{~ms}\left[M_{\text {accuracy }}=57 \%\right.$; $t(14)=5.26, p<.001, r=.82]$, and (f) $33 \mathrm{~ms}\left[M_{\text {accuracy }}=\right.$ $50 \% ; t(14)=.11, p=.91, r=.03]$; see Table 1 .

Effect sizes $(r)$ were then converted to Fisher's $z$ 's to allow for tests of significance between the strength of effect in each of the conditions. This allowed us to compare the accuracy of judgments as a function of exposure time. ${ }^{2}$ The only significant comparisons, however, were against the non-significant effect size for the $33 \mathrm{~ms}$ condition: (a) $10,000 \mathrm{~ms} \quad[Z=2.67, p<.005],($ b) $6500 \mathrm{~ms} \quad[Z=2.59$, $p<.005]$, (c) self-paced [ $Z=3.02, p<.002]$, (d) $100 \mathrm{~ms}$ $[Z=3.38, p<.001]$, and (e) $50 \mathrm{~ms}[Z=2.61, p<.005]$. Null effects between the comparisons for the other conditions (all $p$ 's $>.21$ ) indicate that accuracy was not attenuated by exposure time. Rather, participants were just as accurate at $50 \mathrm{~ms}$ as they are at $10,000 \mathrm{~ms}$. The only point at which scores for accuracy significantly differed was when the effect was no longer significant at $33 \mathrm{~ms}$.

Given that base rates for sexual orientation in society are much greater for heterosexuals than for homosexuals, many participants showed a bias in judging sexual orienta-

\footnotetext{
${ }^{1}$ Mean judgment time for the self-paced condition was about $1500 \mathrm{~ms}$. 2 Rosenthal and Rosnow (1984, p. 372) provide a formula for measuring differences in effect sizes: Fisher's $z=\frac{1}{2} \times \log _{e}(r)$ and $Z=\frac{z_{1}-z_{2}}{\sqrt{\frac{1}{N_{1}-3}+\frac{1}{N_{2}-3}}}$ for both effect sizes ( $r_{1}$ and $r_{2}$, respectively) in which $N_{1}$ and $N_{2}$ correspond to the respective samples. $Z$ refers to the standard normal.
} 
Table 1

Summary statistics for Studies $1 \mathrm{~A}$ and $1 \mathrm{~B}$

\begin{tabular}{|c|c|c|c|c|c|c|c|c|c|c|}
\hline & $M_{\text {Hits }}$ & $M_{\mathrm{FA}}$ & $M_{\% \text { Correct }}$ & $S E_{\% \text { Correct }}$ & $t_{\% \text { Correct }}$ & $r_{\% \text { Correct }}$ & $M_{A^{\prime}}$ & $S E_{A^{\prime}}$ & $t_{A^{\prime}}$ & $r_{A^{\prime}}$ \\
\hline \multicolumn{11}{|l|}{ Study 1A } \\
\hline $33 \mathrm{~ms}$ & .28 & .28 & .50 & .01 & .11 & .03 & .49 & .03 & .32 & .09 \\
\hline $50 \mathrm{~ms}$ & .39 & .26 & .57 & .01 & $5.26^{* * *}$ & .82 & .62 & .02 & $5.61^{* * *}$ & .83 \\
\hline $100 \mathrm{~ms}$ & .49 & .26 & .62 & .01 & $7.73^{* * *}$ & .90 & .69 & .02 & $8.96^{* * *}$ & .92 \\
\hline Self-paced & .56 & .31 & .62 & .02 & $6.47^{* * *}$ & .87 & .70 & .03 & $7.22^{* * *}$ & .89 \\
\hline $6500 \mathrm{~ms}$ & .49 & .33 & .58 & .02 & $5.22^{* * *}$ & .81 & .64 & .03 & $5.41^{* * *}$ & .82 \\
\hline $10,000 \mathrm{~ms}$ & .46 & .25 & .60 & .02 & $5.43^{* * *}$ & .82 & .67 & .03 & $6.08^{* * *}$ & .85 \\
\hline \multicolumn{11}{|l|}{ Study 1B } \\
\hline $50 \mathrm{~ms}$, full set & .38 & .34 & .52 & .01 & $2.87^{* *}$ & .61 & .54 & .01 & $3.13^{* *}$ & .64 \\
\hline $50 \mathrm{~ms}$, reduced set & .38 & .34 & .52 & .01 & $2.29^{*}$ & .52 & .54 & .02 & $2.42^{*}$ & .54 \\
\hline
\end{tabular}



Presentation Time (ms)

Fig. 1. Mean accuracy by percent with standard error for each exposure duration. The $x$-axis crosses at chance accuracy (50\%). Separate lines indicate the uncorrected accuracy rates (mean percent correct), and corrected accuracy rates (response bias eliminated using $A^{\prime}$ ). Ability to accurately classify sexual orientation is consistently above chance for stimulus presentations $50 \mathrm{~ms}$ and longer, whereas accuracy falls to chance at $33 \mathrm{~ms}$. ${ }^{*} 1500 \mathrm{~ms}$ represents the self-paced condition, see Footnote 1.

tion (i.e., they were often more likely to indicate that targets were heterosexual). This bias may have exaggerated the accuracy observed among these data. To correct for response bias, we used the signal-detection statistic $A^{\prime}$ (Rae, 1976), which provided a more conservative estimate of accuracy. Judgments of homosexuality were arbitrarily chosen to represent signal and judgments of heterosexuality as noise. ${ }^{3}$ Thus, hits corresponded to correct identifications of homosexuals, whereas false-alarms corresponded

\footnotetext{
${ }^{3}$ Note that the assignment of signal and noise does not change the result. Specifically, if the analyses are conducted with identifications of straight targets as signal and gay targets as noise, $A^{\prime}$ remains the same. Hence, the assignment of signal and noise is arbitrary.
}

to misidentifications of heterosexuals as homosexual. Given that $A^{\prime}$ ranges between 0 and 1 and describes the area under the ROC curve (i.e., the amount of signal), $A^{\prime}$ scores are functionally equivalent to measures of accuracy by percent. Removing bias resulted in higher levels of accuracy (these data are plotted in Fig. 1): (a) $10,000 \mathrm{~ms}$ $\left[M_{A^{\prime}}=.67 ; t(14)=6.08, p<.001, r=.85\right]$, (b) $6500 \mathrm{~ms}$ $\left[M_{A^{\prime}}=.64 ; t(14)=5.41, p<.001, r=.82\right]$, (c) self-paced $\left[M_{A^{\prime}}=.70 ; \quad t(14)=7.22, \quad p<.001, \quad r=.89\right], \quad(\mathrm{d}) \quad 100 \mathrm{~ms}$ $\left[M_{A^{\prime}}=.69 ; \quad t(14)=8.96, \quad p<.001, \quad r=.92\right], \quad(\mathrm{e}) \quad 50 \mathrm{~ms}$ $\left[M_{A^{\prime}}=.62 ; t(14)=5.61, p<.001, r=.83\right]$, and (f) $33 \mathrm{~ms}$ $\left[M_{A^{\prime}}=.49 ; t(14)=.32, p=.75, r=.09\right]$. In addition, the corrected scores were significantly greater than the uncorrected frequencies $[t(5)=4.26, p<.01, r=.89]$ and male 
and female participants performed equally well, regardless of whether accuracy was uncorrected $[t(88)=.08, p=.94$, $r<.01]$ or corrected $[t(88)=.10, p=.92, r=.01]$.

Similar to above, comparisons of effect sizes showed no differences between conditions where sexual orientation could be accurately discerned (i.e., $50 \mathrm{~ms}$ and greater). In addition, effect sizes for the conditions in which sexual orientation was judged accurately were significantly greater than the effect size for the $33 \mathrm{~ms}$ condition: (a) $10,000 \mathrm{~ms}$ $[Z=2.76, p<.003]$, (b) $6500 \mathrm{~ms}[Z=2.53, p<.006]$, (c) self-paced $[Z=3.11, p<.001],($ d) $100 \mathrm{~ms} \quad[Z=3.57$, $p<.001]$, and (e) $50 \mathrm{~ms}[Z=2.60, p<.005]$. Hence, these analyses permit the same conclusion as that with the uncorrected data: there is no benefit or cost in judgmental accuracy as a function of exposure time.

Since the current study used images from personal advertisements, differences may have been due to the tailoring of the advertisements to different audiences: gay men vs. heterosexual women. Two research assistants coded the contents of 306 gay and 276 straight personal advertisements obtained from the same sources as the photos $\left(R^{\mathrm{SB}}=.989\right)$. Self-descriptive adjectives were recorded for each advertisement and subsequently categorized as stereotypically masculine or feminine. Straight men described themselves more often as feminine and warm $(Z=15.72$, $p<.001)$, whereas gay men more often described themselves using adjectives connoting masculinity and dominance $(Z=2.58, p<.01)$. Hence, both gay and straight men in personal advertisements described themselves as counter-stereotypical. It is therefore unlikely that a selfpresentation bias facilitated the observed effects.

\section{Discussion}

Study 1A demonstrates that sexual orientation can be perceived from brief exposures to men's static faces. Accuracy in judging sexual orientation was above chance for durations as fast at $50 \mathrm{~ms}$. Furthermore, additional time did not significantly increase accuracy for longer durations, including when exposure was self-paced. These findings indicate that rapid perceptions of faces provide enough information to make categorical social judgments just as accurately as when duration of exposure is not restricted.

\section{Study 1B}

Although the results from coding the contents of the personal advertisements suggest that the results of the previous study were not biased by self-presentation, Study 1B controlled more tightly for self-presentation by using a different target sample.

\section{Method}

Fifteen undergraduates ( $n=6$ females) made judgments of images downloaded from www.facebook.com. All of the images came from the same private university in the north- east U.S. Targets in the images were relatively homogeneous in age, socioeconomic status, geography, style of dress/appearance, and level of education. The homosexual and heterosexual groups were approximately matched for racial composition, as well.

The primary advantage of using facebook.com is that it allows individuals to post electronic photo albums, over and above the primary image that one chooses to present of oneself on his or her profile (the latter would be equivalent to the personals photos used in Study 1A). In these albums, individuals will typically post pictures of themselves with their friends, pictures of parties or group outings, and even scenic travel photos. When individuals upload images into their albums, they have the option of providing a title for the photo, a description of the photo, and the opportunity to name others who might be present in the photograph. And when an individual is labeled as being in a photograph, a link is created to that person's profile (provided that they, too, are a facebook.com user) without necessarily obtaining his or her consent to post the photo. When users then view the individual's profile on facebook.com, they will see two types of photos linked to the person's profile: photos posted by the individual, and photos posted by other users ("tagged by others"). Here we used the latter photos. Thus, by using photos of gay and straight individuals that they themselves did not post, we were able to remove the influence of self-presentation and much of the potential selection bias that may be present in photos from personal advertisements.

We performed a search for men's profiles indicating that they were interested in other men for romantic or sexual purposes. Of these profiles, we randomly selected a subset of those persons who had photos of them "tagged by others." A similar procedure was followed for choosing heterosexuals' images, as well. Once a large corpus of photos had been gathered, images were selected and prepared using the same procedures as in Study 1A. This resulted in a total of 160 photos ( 80 homosexual), a set almost twice as large as that used in Study 1A. To exercise further control, we also removed hairstyles from the images using Adobe Photoshop. Previous work in the perception of gender has indicated that hairstyle is a particularly salient cue to distinguishing between men and women (e.g., Roberts \& Bruce, 1988). Given that homosexuals are often considered simply as gender incongruent (e.g., Johnson, Gill, Reichman, \& Tassinary, 2007), differences in hairstyle might be influencing perceptions of the internal features of the targets' faces (see Maclin \& Malpass, 2001).

Procedures were identical to those for the $50 \mathrm{~ms}$ condition above. The $50 \mathrm{~ms}$ condition was chosen because it provides the critical threshold at which judgments of sexual orientation appear to be above chance.

\section{Results and discussion}

Findings replicated those of Study 1A. Participants were significantly greater than chance in judging male sexual ori- 
entation: $M_{\text {accuracy }}=52 \% ; t(14)=2.87, p<.015, r=.61$. Again, accuracy increased when scores were corrected for response bias $\left[M_{A^{\prime}}=.54 ; t(14)=3.13, p<.01, r=.64\right]$ and there were no differences based on participant sex: $t(13)=1.05, p=.31, r=.28$. Although the photos were not posted by the targets themselves, there remains some possibility of presentational bias by the posting targets' friends and acquaintances. To address this, we reanalyzed the data only including photos where the target was not the central figure in the image; i.e., at least one other person was present in the photo. Thus, any intention to present an exaggerated representation of the target (e.g., a photo caricaturing the target's sexual orientation) would be diffused by the poster's attention to the other individuals in the photos. This subset consisted of 69 gay and 64 straight targets. Again, participants' accuracy was significantly better than chance in judging sexual orientation: $M_{\text {accuracy }}=52 \%$; $t(14)=2.29, \quad p<.05, \quad r=.52 ; \quad M_{A^{\prime}}=.54 ; \quad t(14)=2.42$, $p<.03, r=.54$.

Effect sizes for the $50 \mathrm{~ms}$ conditions were compared across the two sets of targets (i.e., Study 1A vs. Study 1B) and this analysis showed that effects in Study 1A did not significantly differ from those in Study 1B for both the uncorrected $(Z=1.02, p=.16)$ and the corrected $(Z=1.02, p=.15)$ data. Thus, the previously observed effect of accurate judgments of sexual orientation at $50 \mathrm{~ms}$ replicated with a more controlled target sample. As the results from the two studies did not significantly differ, we can conclude that images self-selected for personal advertisements do not significantly differ from images selected and posted by others, at least in terms of the perceptibility of male sexual orientation.

\section{General discussion}

Exposure to a face for as little as $50 \mathrm{~ms}$ provides enough information to judge male sexual orientation with abovechance accuracy. Recent findings have indicated that there is consensus, or agreement among perceivers, in their judgments of personality characteristics from glimpses of the face (Bar et al., 2006; Willis \& Todorov, 2006). Similar to this work, we also show here that increased exposure time does not affect the accuracy of judgments from the face. Distinct from this work, however, we extend these effects beyond consensus to the actual accuracy of judgments (see Kenny, 1991, for discussion of consensus vs. accuracy). Further, the speed of exposure time was twice as fast as that observed for consensual judgments of personality and attractiveness, and our use of a backward mask allowed for a more precise indication of exposure time (cf., Willis \& Todorov, 2006).

Evolutionary psychology would suggest that one reason for the ability to distinguish male sexual orientation might be due to the implications for mate selection (see Miller \& Todd, 1998; Thornhill \& Gangestead, 1996). It seems reasonable to suspect that gay men would possess an enhanced capacity for accurately perceiving other gay men because of sexual opportunities. Similar to gay men, it may be advantageous for heterosexual women to parse male sexual orientation to determine the boundaries of their opportunities for mates. It is also possible that heterosexual men may be motivated to attend to the sexual orientations of other men as a means of assessing their competition for mates (Wright \& Sladden, 2003) and in jockeying the social dominance hierarchy (Mazur, 2005). Thus, even though information about male sexual orientation may serve different functions, one can speculate about the motivational function such information might provide for different groups. Replication of the current work with female targets may help to elucidate these possible motivations. Indeed, at present we are limited to drawing conclusions only about male targets.

Despite the unique motivations of each of the above groups to preferentially encode male sexual orientation, it is also possible that there is nothing special about sexual orientation at all and that the current findings simply document the speed and efficiency of accurate person perception, more generally. Considering the ease with which we perceive and categorize perceptually obvious groups and the short durations necessary to reach consensus about the emotions and personality traits of others, it may merely be that we are equipped with a highly efficient capacity for perceiving others. As such, particular motivations regarding mating, honed over human evolution, may not be necessary to precipitate the effects observed in the current work. Rather, the rapid and accurate perception of male sexual orientation may be just another symptom of a fast and efficient cognitive mechanism for perceiving the characteristics of others.

Finally, the finding that male sexual orientation can be accurately perceived in such a short period of time is striking. Although previous work has shown that "thin slices" of behavior are remarkably rich in providing information about people, none have sliced as thin as $50 \mathrm{~ms}$ (Ambady, Bernieri, \& Richeson, 2000). In addition, the use of naturalistic images, as opposed to actors, extends previous work examining consensus in impression formation (cf., Bar et al., 2006; Willis \& Todorov, 2006).

But despite the ecological validity of these effects, one limitation of the present research concerns whether male sexual orientation would remain perceptible if targets attempted to conceal their sexual orientation. As male sexual orientation is a stigmatized identity, it is reasonable to suspect that male homosexuals oftentimes exert the effort to pass as straight (see Goffman, 1963; Yoshino, 2006). It therefore remains an interesting question as to whether accuracy in detecting male sexual orientation might vary as a function of targets' intentions to express or conceal their sexual orientation.

Moreover, the current data are not in a position to suggest whether sexual orientation is manifest because of biological versus cultural differences in appearance. Similarly, we do not attempt to speculate about which features may be distinctive between heterosexual and homosexual men. 
Rather, we know from previous work that the face is a very rich resource of nonverbal information (see Zebrowitz, 1997) and, thus, future studies may work to address what information is extracted from the face in delineating sexual orientation.

In conclusion, the boundary condition for judging male sexual orientation from static pictures of the male face seems to be $50 \mathrm{~ms}$. Brief exposures to the face thus communicate considerable information that is rapidly and efficiently processed by the social perceiver.

\section{References}

Ambady, N., Bernieri, F. J., \& Richeson, J. A. (2000). Toward a histology of social behavior: Judgmental accuracy from thin slices of the behavioral stream. Advances in Experimental Social Psycology, 32, 201-271.

Ambady, N., Hallahan, M., \& Conner, B. (1999). Accuracy of judgments of sexual orientation from thin slices of behavior. Journal of Personality and Social Psychology, 77, 538-547.

Ambady, N., \& Rosenthal, R. (1992). Thin slices of expressive behavior as predictors of interpersonal consequences: A meta-analysis. Psychological Bulletin, 111, 256-274.

Bar, M., Neta, M., \& Linz, H. (2006). Very first impressions. Emotion, 6, 269-278.

Berry, D. S., \& McArthur, L. Z. (1985). Some components and consequences of a babyface. Journal of Personality and Social Psychology, 48, 312-323.

Carroll, L., \& Gilroy, P. J. (2002). Role of appearance and nonverbal behaviors in the perception of sexual orientation among lesbians and gay men. Psychological Reports, 91, 115-122.

Eberhardt, J. L., Goff, P. A., Purdie, V. J., \& Davies, P. G. (2004). Seeing Black: Race, crime, and visual processing. Journal of Personality and Social Psychology, 87, 876-893.

Gaudio, R. P. (1994). Sounding gay: Pitch properties in the speech of gay and straight men. American Speech, 69, 30-57.

Goffman, E. (1963). Stigma: Notes on the management of spoiled identity. Englewood Cliffs, NJ: Prentice-Hall.

Johnson, K. L., Gill, S., Reichman, V., \& Tassinary, L. G. (2007) Swagger, sway, and sexuality: Judging sexual orientation from body motion and morphology. Journal of Personality and Social Psychology, 93, 321-334.
Kenny, D. A. (1991). A general model of consensus and accuracy in interpersonal perception. Psychological Review, 98, 155-163.

Maclin, O. H., \& Malpass, R. S. (2001). Racial categorization of faces: The ambiguous race face effect. Psychology, Public Policy, and Law, 7, 98-118.

Macrae, C. N., \& Bodenhausen, G. V. (2000). Social cognition: Thinking categorically about others. Annual Review of Psychology, 51 , 93-120.

Macrae, C. N., \& Martin, D. (2007). A boy primed Sue: Feature-based processing and person construal. European Journal of Social Psychology, 37, 793-805.

Mason, M. F., Tatkow, E., \& Macrae, C. N. (2005). The look of love: Gaze shifts and person perception. Psychological Science, 16, 236-239.

Mazur, A. (2005). Biosociology of dominance and deference. New York: Rowman \& Littlefield.

Miller, G. F., \& Todd, P. M. (1998). Mate choice turns cognitive. Trends in Cognitive Sciences, 2, 190-198.

Nicholas, C. L. (2004). Gaydar: Eye-gaze as identity recognition among gay men and lesbians. Sexuality \& Culture, 8, 60-86.

Rae, G. (1976). Table of A'. Perceptual and Motor Skills, 42, 98.

Richeson, J. A., \& Trawalter, S. (2005). On the categorization of admired and disliked exemplars of admired and disliked racial groups. Journal of Personality and Social Psychology, 89, 517-530.

Roberts, T., \& Bruce, V. (1988). Feature saliency in judging the sex and familiarity of faces. Perception, 17, 475-581.

Rosenthal, R., \& Rosnow, R. L. (1984). Essentials of behavioral research: Methods and data analysis. New York: McGraw Hill.

Shelp, S. (2002). Gaydar: Visual detection of sexual orientation among gay and straight men. Journal of Homosexuality, 44, 1-14.

Smyth, R., Jacobs, G., \& Rogers, H. (2003). Males voices and perceived sexual orientation: An experimental and theoretical approach. Language in Society, 32, 329-350.

Thornhill, R., \& Gangestead, S. W. (1996). The evolution of human sexuality. Trends in Ecology \& Evolution, 11, 98-102.

Willis, J., \& Todorov, A. (2006). First impressions: Making up your mind after a 100- ms exposure to a face. Psychological Science, 17, 592-598.

Wright, D. B., \& Sladden, B. (2003). An own gender bias and the importance of hair in face recognition. Acta Psychologica, 114, 101-114.

Wright, D. B., \& Stroud, J. N. (2002). Age differences in lineup identification accuracy: People are better with their own age. Law \& Human Behavior, 26, 641-654.

Yoshino, K. (2006). Covering: The hidden assault on our civil rights. New York: Random House.

Zebrowitz, L. A. (1997). Reading faces: Window to the soul? Boulder, CO: Westview. 